\title{
Why are losses from trade unlikely?
}

\author{
Igor Bykadorov $^{1}$, Alexey Gorn ${ }^{2}$, Sergey Kokovin ${ }^{3}$, Evgeny Zhelobodko ${ }^{4}$
}

\begin{abstract}
Examining a standard monopolistic competition model with unspecified utility/cost functions, we find necessary and sufficient conditions on their elasticities for welfare losses to arise from trade or market expansion. Two numerical examples explain the losses: excessive or insufficient entry of firms can be aggravated by market enlargement (under unrealistic elasticities).
\end{abstract}

JEL Codes: F12, L13

Keywords: Market distortions, Trade gains, Variable markups, Demand elasticity.

\section{Introduction}

Gains from trade and large markets are an important issue in monopolistic competition theory (Melitz and Redding [7]), whereas possible losses are less studied, unlike in oligopoly settings (Brander and Krugman [1]). Trying to prove 5 the impossibility of harmful trade, we arrive instead at two counter-examples and a criterion (necessary and sufficient condition) for losses. The objective is to distinguish industries likely or unlikely to be harmed by globalization, by examining properties of their demand and supply functions.

\footnotetext{
${ }^{1}$ National Research University Novosibirsk State University, ul. Pirogova, 2, Novosibirsk 630090, Russia, and National Research University Higher School of Economics, pr. RimskogoKorsakova, 47, St. Petersburg 190068, Russia

${ }^{2}$ Bocconi University, Via Roentgen, 1, Milano 20136, Italy

${ }^{3}$ National Research University Higher School of Economics, pr. Rimskogo-Korsakova, 47, St. Petersburg 190068, Russia, and National Research University Novosibirsk State University, ul. Pirogova, 2, Novosibirsk 630090, Russia

${ }^{4}$ In memoriam, 1973-2013.
} 
This goal requires advanced modelling: variable elasticity of substitution (VES), unspecified preferences and general-form costs. Our setting deviates from Zhelobodko et al. [10] by allowing both convex and concave total cost. This generalization is needed for an important feature: indirect modelling of endogenous technology $(R \& D)$. Indeed, when $R \& D$ is possible, higher output fosters investment in marginal cost reduction, which implies concave cost (Bykadorov 15 et al. [2]).

The main result is condition (9) on utilities/costs, necessary and sufficient for intra-sectoral trade gains or losses in a generalized Dixit-Stiglitz-Krugman model. In addition, two numerical examples demonstrate that this requirement is plausible, i.e., compatible with other reasonable properties of preferences and costs. Therefore both directions of market distortion shows as theoretically possible: excessive or insufficient entry can be aggravated by market growth. However, utilities/costs that satisfy (9) are uncommon, and the related discussion shows why trade losses are unlikely in the real economy.

\section{Model}

The model exposition follows Zhelobodko et al. [10, to ease comparison. Our closed economy exhibits monopolistic competition under unspecified additive utility and cost functions, with variable marginal costs/elasticities. The only production factor is labor, supplied inelastically by $L$ identical consumers/workers. A single sector involves an endogenous interval $[0, N]$ of identical firms producing varieties, one variety per firm.

Each consumer maximizes utility in the form

$$
U=\int_{0}^{N} u\left(x_{i}\right) d i \rightarrow \max _{X \geq 0}, \text { s.t. } \int_{0}^{N} p_{i} x_{i} d i \leq 1 .
$$

Here $X=\left(x_{j}\right)_{j \leq N}$ is a function, $x_{i} \equiv x(i)$ denotes consumer's consumption of $i$ th variety, $p_{i}$ is the price, $w \equiv 1$ is wage, index ${ }_{i}$ everywhere replaces parentheses (i). As in Zhelobodko et al. [10, we use the elasticity operator $\mathcal{E}_{g}(z) \equiv \frac{z g^{\prime}(z)}{g(z)}$ defined for any function $g$, and the Arrow-Pratt concavity operator $r_{g}(z) \equiv$ $35-\frac{z g^{\prime \prime}(z)}{g^{\prime}(z)}=-\mathcal{E}_{g^{\prime}}(z)$. 
For existence, uniqueness and symmetry of the equilibrium, we make the following weak restrictions on utility (Zhelobodko et al. [10, Mrzov and Neary [9]). At some zone $[0, \check{z})$ of possible equilibria $(\check{z} \leq \infty)$, the elementary utility function $u(\cdot)$ is thrice differentiable -increasing $\left(u^{\prime}(z)>0\right)$, strictly concave ${ }_{40}\left(u^{\prime \prime}(z)<0\right)$, normalized $(u(0)=0)$-and its main characteristics behave as $r_{u}(z) \in[0,1), r_{u^{\prime}}(z)<2 \forall z \in[0, \check{z})$.

Then the first-order condition (FOC) with a Lagrange multiplier $\lambda$ entails the inverse demand function $\mathbf{p}$ for any variety $i$ :

$$
\mathbf{p}\left(x_{i}, \lambda\right)=\frac{u^{\prime}\left(x_{i}\right)}{\lambda}
$$

The marginal utility of income $\lambda$ serves as the single market aggregate.

Each producer faces some total cost function $C(q)$ depending upon output $q \equiv L x$, perceives function $\mathbf{p}$ and $\lambda$ as given, and maximizes profit

$$
\pi(x, \lambda) \equiv \frac{u^{\prime}(x)}{\lambda} x L-C(L x) \rightarrow \max _{x \geq 0}
$$

(Here, choice of maximizers $x, q$ or $p$ brings an equivalent result, and the firm's index $i$ is dropped by symmetry.) Denoting revenue $R(x, \lambda, L) \equiv u^{\prime}(x) x L / \lambda$, we can formulate the FOC in usual terms of marginal revenue and marginal cost: $\frac{d}{d x} R(x, \lambda, L)-\frac{d}{d x} C(L x)=0$. The second-order condition (SOC) is

$$
-\frac{d^{2} \pi}{d^{2} x}=-\frac{d^{2}}{d^{2} x} R(x, \lambda, L)+\frac{d^{2}}{d^{2} x} C(L x)>0 .
$$

This assumption justifies symmetry of the equilibrium.

Equilibrium is a bundle $(\bar{x}, \bar{p}, \bar{\lambda}, \bar{N})$ satisfying the utility maximization condition (2); profit maximization FOC and SOC; free-entry and labor market clearing conditions:

$$
\begin{gathered}
R(\bar{x}, \bar{\lambda}, L)-C(L \bar{x})=0, \\
\bar{N} C(L \bar{x})=L .
\end{gathered}
$$

(Upper bar henceforth denotes equilibria.)

Now we can divide each producer's FOC by the free-entry condition to express our equilibrium through the elasticity of revenue $\mathcal{E}_{R}$, the elasticity of 
inverse demand $\mathcal{E}_{\mathbf{p}}(x) \equiv \frac{x}{\mathbf{p}} \cdot \frac{\partial \mathbf{p}(x)}{\partial x} \equiv-r_{u}(x)$ and the cost elasticity $\mathcal{E}_{C}(q) \equiv$ $\frac{q}{C} \cdot \frac{\partial C(q)}{\partial q}:$

$$
\mathcal{E}_{R}(\bar{x}) \equiv 1-r_{u}(\bar{x})=\mathcal{E}_{C}(L \bar{x}) .
$$

45 The equilibrium consumption $\bar{x}$ is determined here, whereas equilibrium prices $\bar{p}$ and mass $\bar{N}$ of firms can be found from the remaining equations. Therefore, each consumer's equilibrium welfare $\bar{U}=\bar{N} u(\bar{x})$ depends indirectly on market size $L$ through the equilibrium magnitudes $\bar{x}(L), \bar{N}(L)$.

Totally differentiating the equilibrium equation (5) w.r.t. population size $L$ and using (4), we express total utility elasticity $E_{\bar{U} / L}$ at equilibrium through

other total elasticities $E_{\bar{N} / L} \equiv \frac{L}{N} \cdot \frac{d \bar{N}}{d L}, E_{\bar{x} / L} \equiv \frac{L}{\bar{x}} \cdot \frac{d \bar{x}}{d L}$ and partial elasticity $\mathcal{E}_{u} \equiv \mathcal{E}_{u}(\bar{x}) \equiv \frac{z}{u(z)} \cdot \frac{\partial u(z)}{\partial z}$ as follows:

$$
E_{\bar{U} / L} \equiv \frac{L}{\bar{U}} \cdot \frac{d \bar{U}}{d L}=E_{\bar{N} / L}+\mathcal{E}_{u} \cdot E_{\bar{x} / L} .
$$

The SOC for profit maximization at equilibrium is

$$
S O C \equiv r_{u}^{\prime}(\bar{x}) \cdot \bar{x}+\mathcal{E}_{C}^{\prime}(L \bar{x}) \cdot L \bar{x}>0 .
$$

(Proofs are in Bykadorov et al. [3].)

\section{Losses from market size}

Lemma. The local effect of a growing market on welfare can be expressed in elasticities (taken at the equilibrium values) as follows:

$$
E_{\bar{U} / L}=\left(1-\mathcal{E}_{u}\right)-\frac{\bar{x}^{2}}{\mathcal{E}_{u}} \cdot \frac{\mathcal{E}_{u}^{\prime} \cdot r_{u}^{\prime}}{S O C}=r_{u}+\frac{L \bar{x}^{2}}{\mathcal{E}_{u}} \cdot \frac{\mathcal{E}_{u}^{\prime} \cdot \mathcal{E}_{C}^{\prime}}{S O C}
$$

This lemma enables us to establish the necessary and sufficient condition for "harmful trade" through the following claims, each highlighting some aspect of market distortion.

Proposition. Consider an equilibrium $\bar{x}$ under market size $L_{0}$. Any local welfare reduction caused by a growing market is equivalent to the following 
conditions on utility, revenue and cost elasticities:

$$
E_{\bar{U} / L}<0 \Leftrightarrow \mathcal{E}_{R}^{\prime}(\bar{x})<\mathcal{E}_{C}^{\prime}\left(L_{0} \bar{x}\right) \cdot L_{0}<\mathcal{E}_{R}^{\prime}(\bar{x}) \cdot \frac{r_{u}(\bar{x})}{1-\mathcal{E}_{u}(\bar{x})} .
$$

For a more convenient interpretation, this double inequality can be reformu-

60 lated as follows.

Corollary. (i) [Necessity]. For any welfare reduction two conditions are necessary:

$$
\begin{gathered}
\mathcal{E}_{u}^{\prime}(\bar{x}) \cdot \mathcal{E}_{R}^{\prime}(\bar{x})<0, \\
\mathcal{E}_{u}^{\prime}(\bar{x}) \cdot \mathcal{E}_{C}^{\prime}(L \bar{x})<0 .
\end{gathered}
$$

In particular, under convex cost $\left(\mathcal{E}_{C}^{\prime}>0\right)$, such reduction requires both increasingly elastic revenue (IER) and decreasingly elastic utility (DEU).

(ii) [Sufficiency]. For any utility satisfying inequality (10) at some $\bar{x}$ under given $L_{0}$, one can find a cost function $C$ such that $\bar{x}$ is an equilibrium, and welfare locally decreases w.r.t. $L$ at $L_{0}$. One can find also another cost function $\tilde{C}$ that makes welfare locally increasing.

Discussion. Under properties (9), (10) and (11) holding globally, these claims are easily extended from infinitesimal changes in population and welfare $\left(\frac{d \bar{U}}{d L}\right)$ onto global ones $\left(\frac{\Delta \bar{U}}{\Delta L}\right)$.

70 Why are equilibria satisfying all conditions $(9),(10)$ and $(11)$ unlikely? Property $\mathcal{E}_{R}^{\prime}(x) \equiv-r_{u}^{\prime}(x)<0$ is called decreasingly elastic revenue (DER), being equivalent to increasingly elastic (strictly subconvex) demand (Mrzov and Neary [8). The DER case is called realistic by Krugman [6] and subsequent papers (see Zhelobodko et al. [10]) because it generates decreasing prices under in75 creasing competition; DER is perceived as "Marshall's Second Law of Demand" by Mrzov and Neary [8]. Then, 8 becomes $1>\frac{-\mathcal{E}_{C}^{\prime}(L \bar{x}) \cdot L}{r_{u}^{\prime}(\bar{x})}>\frac{r_{u}(\bar{x})}{1-\mathcal{E}_{u}(\bar{x})}$. To get losses, some $C($.$) must fit this double inequality, compatible only when$ $\mathcal{E}_{u}<1-r_{u}=\mathcal{E}_{R}$, which is problematic. Indeed, finite $u^{\prime}(0)>0$ (chokeprice) and negative $u^{\prime}(\infty)<0$ (satiabile demand) are reasonable assump80 tions that entail $\mathcal{E}_{u}=1, \lim _{x \rightarrow \infty} \mathcal{E}_{u}(x)<0$. Then, $\mathcal{E}_{u}$ generally decreases: 
$0 \geq \mathcal{E}_{\mathcal{E}_{u}} \equiv \mathcal{E}_{R}-\mathcal{E}_{u}:[0, \infty)$, except, maybe, for some interval. For $E_{\bar{U} / L}<0$, the equilibrium must occur in this interval, which is empty for all typical utilities: CARA, HARA, CES, quadratic. Even for specially constructed exotic utilities, the interval is tiny, the freedom of choice in $\sqrt{9}$ being small: $\frac{r_{u}(\bar{x})}{1-\mathcal{E}_{u}(\bar{x})} \approx 1$ (see our examples). Moreover, reasonable cost specifications $C \equiv f+c(f) q$ where function $c(f)$ decreases not too quickly, yield $\mathcal{E}_{C}^{\prime}>0$, which is incompatible with (9) when $\mathcal{E}_{R}^{\prime}(x)<0$. Summarizing, too many very stringent conditions must hold simultaneously to generate losses from trade.

Literature and interpretation. Elasticities' role in welfare is known since Dixit and Stiglitz [4: under linear cost $(C \equiv f+c q)$, CES yields optimum, whereas firms' entry is socially excessive in DEU case $\left(\mathcal{E}_{u}^{\prime}<0\right)$ but insufficient under IEU $\left(\mathcal{E}_{u}^{\prime}>0\right)$. Under market expansion, such a distortion diminishes under DER, and the consumer enjoys a "double benefit" from growing variety and decreasing prices (Krugman [6]). This claim is generalized to convex cost in Zhelobodko et al. [10, to costly trade in Mrzov and Neary [9], and to firms' heterogeneity in Dhingra and Morrow [5], where functions $r_{u}(x)$ and $1-\mathcal{E}_{u}(x)$ are called "markup" and "social markup," respectively. These two are "aligned" when both increase (DER, DEU) or both decrease (IER, IEU); when preferences are aligned, market expansion increases welfare. In economic terms, "the market maximizes markup but the social planner pursues social markup; when these goals are aligned, demand shifts alter private and social markups in the same direction" (Dhingra and Morrow [5]).

We have shown that aligned preferences serve also as a necessary condition for welfare gains, in the sense of "gains occuring under any cost function." Another contribution is non-linear cost, e.g., endogenous technology. Though according to Dhingra and Morrow [5] "these are the demand-side elasticities that determine how resources are misallocated and when ... market expansion provides welfare gains" but (9) shows that costs also matter.

The only mathematical example of harmful trade in the New Trade theory that we know - we have learned from Peter Neary in private talk, 2013. It 
exploits a non-normalized utility, e.g., $u(x)=\sqrt{x}-a(a>0)$, that violates our assumptions. However, we do not perceive violating normalization as legitimate. Indeed, an arbitrary constant added to the sub-utility should not change all the welfare conclusions from plus to minus. Instead, we provide two legitimate examples.

[Insert Figure here]

Example 1: IER and DEU (excessive entry aggravated, Fig.2.1, left panel)

To demonstrate the possibility of harmful trade, we extend the CARA utility with a linear or polynomial term:

$$
u(x)=\left\{\begin{array}{cc}
1-\exp (-x)+2 x & \text { if } x<2 \\
-\exp (-x)+3 x-0.25 x^{2} & \text { if } x \geq 2
\end{array}\right\} .
$$

Here IER property holds under $x<2$, where all our equilibria lie (interval $x \geq 2$ is constructed only to satisfy our restrictions on $u$ ). Elasticity $\mathcal{E}_{u}(x)=\frac{(2+\exp (-x)) x}{1-\exp (-x)+2 x}$ is computed and plotted by software as the thick orange dotted curve in the figure. Revenue elasticity $\mathcal{E}_{R}(x)=1-r_{u}(x)=\frac{\exp (-x) x}{2+\exp (-x)}$ is painted thick blue. The initial market size is $L_{1}=3.4447$; related cost elasticity $\mathcal{E}_{C}\left(L_{1} x\right)=\frac{L_{1} c x}{f+L_{1} c x}$ is painted dashed thick magenta $(C(q) \equiv f+c q=1+q)$. The equilibrium equation (5) here means the lower of the two intersections between $\mathcal{E}_{R}(x)$ and $\mathcal{E}_{C}\left(L_{1} x\right)$, almost indistinguishable because of almost tangent curves: $\frac{r_{u}(\bar{x})}{1-\mathcal{E}_{u}(\bar{x})} \approx 1 \Rightarrow \mathcal{E}_{C}^{\prime} \approx \mathcal{E}_{R}^{\prime}$ ). This (pink) equilibrium point is $x_{1} \approx 1.98683, U_{1} \approx 2.12396$. When market size expands to $L_{2}=3.446$, we get a new, thin dashed, curve $\mathcal{E}_{C}\left(L_{2} x\right)$, which almost coincides with old curve $\mathcal{E}_{C}\left(L_{1} x\right)$. It brings a new (black) equilibrium point with smaller consumption and utility: $x_{2} \approx 1.96165<x_{1}, U_{2} \approx 2.12389<U_{1}$. The mass of firms increases $\left(N_{1}=0.439149<N_{2}=0.444081\right)$ but insufficiently.

Initially, excessive entry here implies inefficiently high average costs. Subsequently, an increase in $L$ pulls the average cost up, driving the economy further away from optimum. Individual consumption decreases, being insufficiently 
compensated by increasing variety. However, excessive entry is aggravated by market expansion only if cost elasticity is almost tangent to revenue elasticity, as one can understand from $\frac{r_{u}(\bar{x})}{1-\mathcal{E}_{u}(\bar{x})} \approx 1$.

Example 2: DER and IEU (insufficient entry aggravated, Fig.2.1, right panel)

Consider be-power utility

$$
u(x)=\left\{\begin{array}{cl}
0.5 \sqrt{0.125+x}-0.125 x^{3 / 4}+0.125 \cdot 2^{-1 / 4} & \text { if } x \geq 0.1 \\
6.04076 x-28.4021 x^{2} & \text { if } x<0.1
\end{array}\right\} .
$$

All our equilibria belong to $x \geq 0.1$; the initial interval $x \in[0,0.1]$ is constructed to fit our assumption $u(0)=0$. The non-linear cost function is $C(q)=0.2+$ $1451.5 \exp (-1 / \sqrt{q / 7})$. Fig.2.1-right maintains the same colours and legend as the left panel. Under $L_{1}=4.25$, the first equilibrium point is $x_{1} \approx 0.669948, U_{1} \approx$ 3.7994. Increasing the market size to $L_{2}=4.47$, we get another equilibrium point with higher consumption $x_{2} \approx 0.921192>x_{1}$ and smaller utility $U_{2} \approx$ $3.6732<U_{1}$. The mass of firms decreases noticeably: $N_{2}=7.36122<N_{1}=$ $150 \quad 8.28935$.

Unlike the previous example, the initial social distortion takes here the form of insufficient entry (variety) and related low average costs. Then, in response to increasing $L$, variety further decreases, being insufficiently compensated by the consumption hike and thus further departs from optimum. Again, distortion is aggravated by market expansion only when cost elasticity is almost tangent to revenue elasticity (8).

To summarize, losses from trade require several highly stringent conditions to hold simultaneously: increasingly elastic misaligned preferences and quite specific costs. For more definite conclusion, condition (9) should be falsified or confirmed empirically.

Acknowledgments. We are indebted to Jacques-Franois Thisse, Kristian Behrens, Richard Ericson, Mila Kasheeva, Sergey Kichko, Yasusada Murata, Mathieu Parenti, Philip Ushchev and very thorough anonynous referee for 
valuable comments. We gratefully acknowledge financing by Russian government grant 11.G34.31.0059, grant 12-06-00174 from RFBR, grant 11-5231 from EERC.

\section{References}

\section{References}

[1] Brander, A., Krugman, P., 1983. A 'Reciprocal Dumping' Model of International Trade. Journal of International Economics, 15, 313-321.

[2] Bykadorov, I., Kokovin, S., Zhelobodko, E., 2013. Investments in Productivity under Monopolistic Competition: Large 
[6] Krugman, P.R., 1979. Increasing returns, monopolistic competition, and international trade. Journal of International Economics 9(4), 469479.

[7] Melitz, M., Redding, S.J., 2012. Heterogeneous Firms and Trade. NBER WP 18652.

[8] Mrzov, M., Neary, J.P., 2013. Not so demanding: preference structure, firm behavior, and welfare. University of Oxford WP 691.

[9] Mrzov, M., Neary, J.P., 2014. Together at Last: Trade Costs, Demand Structure, and Welfare. American Economic Review 104(5), 298-303.

[10] Zhelobodko, E., Kokovin, S., Parenti, M., Thisse, J.-F., 2012 Monopolistic competition in general equilibrium: Beyond the Constant Elasticity of Substitution. Econometrica 80(6), 27652784 . 

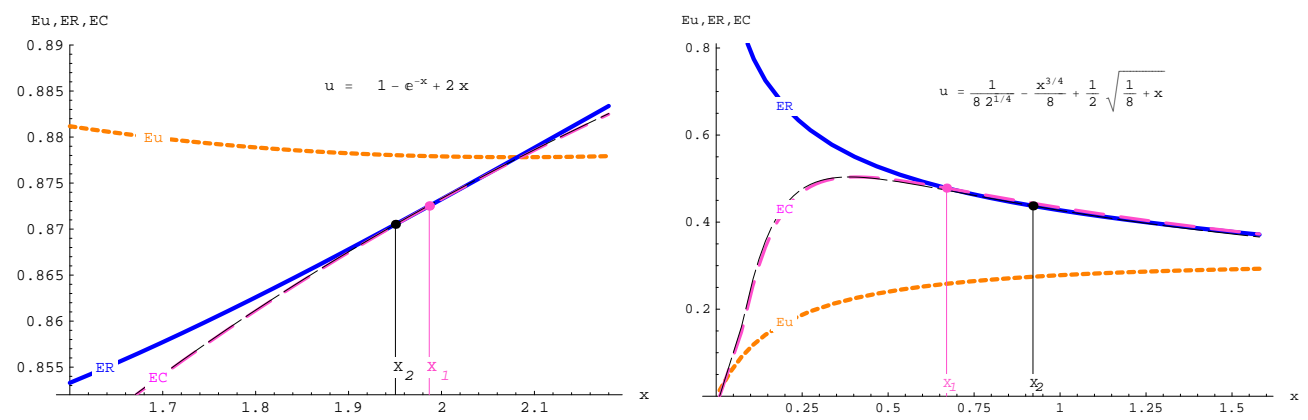

Figure 0.1: Misaligned elasticities and welfare loss: two examples 